All patients showed immediate sphincter relaxation. Two minutes after injection, the mean maximum resting pressure was reduced by $44 \%$ compared with baseline. Bleeding of the anal fissure stopped within 48 hours of injection in all patients. In acute cases, all fissures had healed by the 15-day follow-up examination, whereas chronic fissures healed within 28 days. One patient relapsed after three months and required surgery, but all other patients $(98 \%)$ remained asymptomatic and free of pain during the 14-month followup. There were no reported side effects.

Garrido et al. observe that the efficacy of this treatment was superior to that of other pharmacologic agents for anal fissures. Since this approach also avoids the problems and costs of surgical intervention, they propose that gonyautoxin should be regarded as a superior treatment in this setting.

Original article Garrido R et al. (2005) Gonyautoxin: new treatment for healing acute and chronic anal fissures. Dis Colon Rectum [doi: 10.1007/s10350-004-0893-4]

\section{Long-term survival in pancreatic ductal adenocarcinoma}

Although prognosis is generally very poor in patients with pancreatic ductal adenocarcinoma, long-term survival rates are unclear. A team from Finland has recently studied their nationwide cancer registry data in an attempt to clarify this question.

The registry collects data on cancer cases throughout the Finnish population of 5.1 million people. In the period 1990-1996, 4,922 cases of pancreatic cancer were recorded. Of these, 89 patients survived for at least 5 years; these cases were re-evaluated by the investigators.

Almost half of the long-term survivors had tumors other than pancreatic ductal adenocarcinoma. Of the remaining 44 patients, histologic confirmation of pancreatic cancer was lacking in 18 cases. There were only 26 cases, therefore, of histologically proven pancreatic ductal adenocarcinoma. Re-evaluation of the corresponding slides or paraffin blocks by Carpelan-Holmström and colleagues, however, confirmed the diagnosis in only 10 cases. With the exception of two cases where histologic specimens were unavailable, the remainder were considered by the investigators to have been wrongly diagnosed.

Given that $90 \%$ of pancreatic cancers are pancreatic ductal adenocarcinoma, say the authors, the true 5-year survival rate for patients with this tumor was only $0.2 \%$. They conclude that higher long-term survival rates probably stem from false diagnoses, and encourage thorough histopathologic review of all patients with pancreatic cancer.

Original article Carpelan-Holmström M et al. (2005)

Does anyone survive pancreatic ductal adenocarcinoma? A nationwide study re-evaluating the data of the Finnish Cancer Registry. Gut 54: 385-387

\section{Colorectal cancer survival among European and US patients}

Colorectal cancer survival rates are markedly lower in Europe than in the US. Ciccolallo et al. have explored the reasons behind this variation using population-based registry data.

Ten European cancer registries provided details of 2,492 consecutive cases of primary invasive adenocarcinoma of the large bowel diagnosed in 1990-1991. Data on a further 11,191 cases were extracted from the seer database in the US. In addition to details of surgical treatment, the information included disease stage and number of examined lymph nodes as a determinant of staging accuracy.

As expected, 3-year relative survival was higher in the US (69\%) than in Europe (57\%). In all European registries except Modena and Eindhoven, the crude relative excess risk of death was significant, ranging from 1.26 to 2.21. Patients in the US were more likely to be diagnosed with localized disease and to undergo surgical resection than their European counterparts (54\% vs $48 \%$ and $92 \%$ vs $85 \%$, respectively). In addition, examination of 12 or more lymph nodes was more common in the US. It should be noted that data from the individual European registries varied widely; the rates of localized disease among patients from the Dutch registries, for example, were higher than those in the US.

The study highlights the differences in the diagnosis and treatment of colorectal cancer in Europe and the US, and indicates that 